International Journal of Wireless \& Mobile Networks ( IJWMN ), Vol.2, No.3, August 2010

\title{
THROUGHPUT IMPROVEMENT OF MOBILE MULTI-HOP WIRELESS NETWORKS
}

\author{
Barbaros Preveze $^{1}$ and Aysel Şafak ${ }^{2}$ \\ ${ }^{1}$ Electronics and Communication Engineering, Çankaya University, Ankara, Turkey \\ b.preveze@cankaya.edu.tr \\ ${ }^{2}$ Electrical and Electronics Engineering, Başkent University, Ankara, Turkey \\ asafakebaskent.edu.tr
}

\begin{abstract}
This paper demonstrates the use of some novel cognitive techniques we propose to improve the throughput of a mobile WIMAX network and minimize the packet losses. We propose a novel method called Orthogonal Frequency Division Most Congested Access First (OFDMCAF), to modify the spectrum sharing technique of (Time Division Multiple Access) TDMA and (Orthogonal Frequency Division Multiple Access) OFDMA and to manage the spectrum access. The heavy multimedia traffic is managed by OFDMCAF with novel spectrum sharing and buffer management methods we propose. By use of novel cognitive methods, real time voice and video packet transmissions have been provided with full success and the packet loss rate of data packets has been minimized. The simulation results are confirmed by comparing them with results of other publications and comparing with results of packet loss calculations we made. The results are usable in designing unicast mobile multimedia network with improved system throughput.
\end{abstract}

\section{KEYWORD}

Throughput, Cognitive, WIMAX, Multimedia, Routing

\section{INTRODUCTION}

In the cellular network that we consider, all the relay nodes try to send their packets in different signal shapes such as video, voice or data to random destination points traversing a calculated multi-hop path. So the nodes have to share the spectrum among each other. In this system OFDMA and TDMA [1, 2, 3] based spectrum sharing techniques are used.

We have developed an event-driven simulation program using MATLAB in which, the movements, locations and buffer states of the nodes, the organization of the packets in the buffer of each node, selected paths for each packet, data generation/transmission rate and the instant throughput values are all observable from the screen. The parameter set in the simulation program can be changed before the simulation run, so the simulation can run for any number of nodes, any video or voice packet generation/transmission rate or packet generation rate.

In our simulation, $\mathrm{N}$ independent and uniformly distributed mobile relay nodes are considered to communicate with each other in a cell structure. It is assumed as in [4] that, all relay nodes continuously have real time CBR (constant bit rate) video and voice 
conversations with random relay nodes in the network. It is also assumed for BE (best effort) that we have non-stop packet generation at data queues of relay nodes for which transmission rate changes according to network conditions.

In cognitive systems, the nodes Observe the environment, Orient the information of locations, buffer statuses, packet loss ratios, average hop counts, unsuccessful transmission trial rates etc., then they Decide what to do and Act (OODA (ObserveOrient-Decide-Act) loop). The parameters in cognitive networks are updated based on the active monitoring of radio frequency spectrum, user behavior and network state [5].

By this study, the throughput improvement of a mobile multi-hop WIMAX system including heavy multimedia packet traffic is provided. For evaluation of the results, we run the simulation for 1000 OFDMA frames for absence case of each algorithm where all the nodes generate and transmit their video, voice and data packets to their calculated next nodes in each OFDMA frame. The route at any instant is determined by use of Fastest Path routing Algorithm in [6].

The buffer sizes in the simulations are used as calculated in Section 3.4. The packets in the buffers are controlled according to the algorithm declared in Section 4.1.3. In Section 6.2, the results for varied $\mathrm{N}$ values we had, are compared with the results of asymptotic analysis made in literature. The improvements on network throughput by novel spectrum access and buffer management approaches are illustrated. Note that the non improved results of novel proposed methods match with the results evaluated in the literature and the results can also be evaluated using any other popular event- driven network simulator in the area by providing the same parameter values used in the simulation.

\section{Determination Of Maximum Spectral Usage In Multi-Hop MOBILE WIMAX (MMW) NETWORK}

The parameter values in Table 1 and formulations in eq. 1-12 are selected according to calculations made in [1] and the standards [1, 3].

$$
\begin{gathered}
\text { Frames per second }(\mathrm{FPS})=1 / \mathrm{FL} \\
\text { Bits per symbol }(\mathrm{BPS})=\log _{2}(\mathrm{QAM}) \times \mathrm{NODS} \\
\text { Symbols per frame }(\mathrm{SPF})=\mathrm{FL} / \mathrm{TST} \\
\text { sub - channel data rate in a frame with FEC }(\mathrm{SCDRFWF})=\mathrm{DSPF} \times \mathrm{MAU} \\
\text { sub }- \text { channel data rate in a second with FEC }(\mathrm{SCDRSWF})=\mathrm{SCDRFWF} \times \mathrm{FPS} \\
\text { Minimum Allocatable Unit }(\mathrm{MAU})=\mathrm{BPSWF} / \mathrm{NOS} \\
\text { Sampling Rate }(\mathrm{SR})=\mathrm{B} \times \mathrm{OSF} \\
\text { Data rate per sec With FEC }(\mathrm{DRPSWF})=\mathrm{FEC} \times \mathrm{DRPS} \\
\text { Data rate per sec. }(\mathrm{DRPS})=\mathrm{SRPS} \times \mathrm{BPS}
\end{gathered}
$$


International Journal of Wireless \& Mobile Networks ( IJWMN ), Vol.2, No.3, August 2010

$$
\begin{gathered}
\text { Bits per symbol with FEC }(\text { BPSWF })=\text { FEC } \times \text { BPS } \\
\text { Symbol rate per sec. }(\text { SRPS })=\text { FPS } \times \text { DSPF }
\end{gathered}
$$

sub-channel data rate in a second With FEC $($ SCDRSWF $)=S C D R F W F \times F P S$

The abbreviation NODS in eq. 2, stands for number of Data sub-carriers.

Table 1. System parameters used in the simulation for mobile WIMAX defined by the standards $[1,3]$

\begin{tabular}{|l|l|}
\hline Guard Rate $(\mathrm{GR})=1 / 8$ & Sampling Period $(\mathrm{TS})=1 / \mathrm{FS}=91.4 \mu \mathrm{s}$ \\
\hline Total used Freq. $(\mathrm{FT})=1 / \mathrm{TST}=9.718 \mathrm{kHz}$ & Total symbol Time $(\mathrm{TST})=102.8 \mu \mathrm{s}$ \\
\hline Sampling guard interval(TSG) $=11.4 \mu \mathrm{s}$ & Over Sampling factor $(\mathrm{OSF})=8 / 7$ \\
\hline Frame Length(FL) $=5 \mathrm{~ms}$ & Forward Error Correction $(\mathrm{FEC})=3 / 4$ \\
\hline Frequency spacing $(\mathrm{FS})=10.94 \mathrm{MHz}$ & Sub channel Capacity $(\mathrm{SCC})=0.95 \mathrm{Mbps}$ \\
\hline Data symbols per Frame $(\mathrm{DSPF})=44$ & \begin{tabular}{l} 
Number of sub-channels $(\mathrm{NOS})=30$ \\
\hline Bandwidth $(\mathrm{B})=10 \mathrm{MHz}$
\end{tabular} \\
\hline
\end{tabular}

In the simulation program, at first the maximum possible throughput that the system may provide is determined using the equations and input parameters given in Table 1. Using B (Bandwidth) $=10 \mathrm{MHz}$ (between $2.3 \mathrm{GHz} \& 2.4 \mathrm{GHz}$ ) [3], choosing modulation $=64$ QAM, DTPS $($ Data Packet size) $=150$ bytes, VCPS (Voice Packet size) $=10$ bytes, VDPS (Video Packet size $)=512$ bytes, FL(Frame Length $)=5 \mathrm{~ms}(1 / 0.005=200$ fps) (from eq. 1), FEC (Forward Error Correction) Rate $=3 / 4$, and using rest of the parameters as defined by the standards given in Table 1 or calculating those using the eqs. 1-12. In order to calculate the Maximum spectral usage upper bound, we have,

$$
\begin{aligned}
\text { Bits per symbol }(\mathrm{BPS}) & =6 \text { bits } / \text { subcarrier } \times 720 \text { subcarrier } \\
& =4320 \text { bits }
\end{aligned}
$$

from eq.2. For $\mathrm{NOS}=30, \mathrm{~B}=10 \mathrm{MHz}(\mathrm{FFT}=1024)$, using eq. 6 and eq.10, we have;

$$
\begin{aligned}
\text { Bits per symbol with FEC (BPSWF) } & =\frac{3}{4} \times 4320 \\
& =3240 \text { bits } \\
\text { Minimum Allocatable Unit }(\text { MAU }) & =3240 / 30 \\
& =108 \mathrm{bits}
\end{aligned}
$$

Considering a device is allotted one sub-channel in every frame, there will be 200 frames/sec (from eq. 1) with $\mathrm{DSPF}=44 \mathrm{OFDM}$ data symbols/frame each, which results with 8800 using eq. 11 such that,

$$
\begin{aligned}
& \text { Symbol rate per sec. }(\text { SRPS })=(200 \times 44) \\
& =8800 \text { data symbols } / \text { frame }
\end{aligned}
$$


International Journal of Wireless \& Mobile Networks ( IJWMN ), Vol.2, No.3, August 2010

as the resultant value is also given in Table 1, the value of SCC can be calculated [1] by,

$$
\begin{aligned}
\text { Sub-channel capacity in a frame with } \operatorname{FEC}(\mathrm{SCC}) & =(M A U \times S R P S)) \\
& =108 \text { bits } \times 8800 \text { bits } \\
& =950400 \text { bits }
\end{aligned}
$$

then the Maximum spectrum usage (MSU) can be calculated as in eq.13, by multiplying the sub-channel capacity with the number of sub-channels as;

$$
\begin{aligned}
\text { Max. spectrum usage }(\mathrm{MSU}) & =S C C \times N O S \\
& =950400 \mathrm{bits} / \mathrm{second} \times 30 \\
& =28512 \times 10^{6} \\
& =3564000 \mathrm{bytes} / \mathrm{sec}
\end{aligned}
$$

This upper bound will give us idea about how close to the upper bound we could bring the throughput. The results also match with the results evaluated in [1] and defined by standards [3].

\section{Packet Carriage Of Multi-hop Mobile WiMAX (MMW)}

\subsection{Real time Multimedia Packet Carriage of MMW}

The nodes are considered to generate 50 picture frames per second for video conversation as in [1]. This means 50 of 200 OFDM frames within a sub-channel per second will be used to send video packets. Multiplying the SCC value given in Table 1, by Video Packet Sending Rate (VPSR) of 50/200, we have the video rate as in eq. 14. The value evaluated in eq.14 can be handled by a device using H.264 video codec. Since we have 30 sub-channels in each frame and 200 frames in a sec., video packet size will be calculated as in eq. 15 .

$$
\begin{aligned}
\begin{aligned}
\text { video rate } & =S C C /\left(\frac{1}{V P S R}\right) \\
& =(950400 \mathrm{bits} / \mathrm{sec}) /\left(\frac{1}{50 / 200}\right) \\
& =237600 \mathrm{bits} \Rightarrow 29700 \text { bytes }
\end{aligned} \\
\text { VDPS }=\text { SCDRFWF } \\
=\frac{\frac{\mathrm{MSU}}{\mathrm{NOS}}}{\frac{1}{\mathrm{FL}}}=\frac{\frac{3564000 \mathrm{bytes} / \mathrm{s}}{30}}{200} \Rightarrow 594 \text { bytes }
\end{aligned}
$$

If we consider that, each node in the network is having voice conversation in parallel to video conversation, and $16 \mathrm{kbps}$ voice packets can be considered due to low latency requirements. According to [1], voice packet size is calculated as in eq. 16, where our system has 200 frames in a second with frame length of $5 \mathrm{~ms}$.

$$
\text { VCPS }=\frac{16 \text { kbits }}{200} \Rightarrow 10 \text { bytes }
$$

\subsection{Non-Real Time Data Carriage of MWM}

In order to overcome the heavy traffic conditions, the nodes will use different number of sub-channels (SC) for different data types. Note that, the default number of nodes is 
taken as $\mathrm{N}=6$ in section 6.1 and in the explanations of this paper as in [7], but $\mathrm{N}$ is varied from 4 to 24 in the simulations in Section 6.2. The number of sub-channels allocated for data transmission in a frame, can be calculated for the given parameters by subtracting the total number of sub-channels used by video and voice packets from the total number of sub-channels as in eq. 17. For $\mathrm{N}=6$, we have;

$$
\begin{aligned}
\text { TSFDT } & =(\mathrm{SC}-(\mathrm{TSCFVD}+\mathrm{TSCFVC}) \\
& =(30-(6 \times 1+1 \text { or } 2) \\
& =22 \text { or } 23 \mathrm{SC}
\end{aligned}
$$

In eq. 17; TSFDT is "Total SC for Data Packets", TSCFVD is "Total SC for Video Packets" and TSCFVC is "Total Sc for Voice Packets". The system is always aware of number of SC used by each data type. Since only 1 out of 4 SC is used by each node for video conversations, 1 or $2 \mathrm{SC}$ will be used in a frame by the nodes for video packet transmission. Hence, if $\mathrm{N}=6$, the nodes will transmit their data packets using 22 or 23 sub-channels. The calculated data packet size nearest to chosen packet size for $\mathrm{N}=6$, can be evaluated for minimum bandwidth wastage by;

$$
\begin{aligned}
\text { DTPS } & =\frac{(\mathrm{SCDRFWF})}{\text { floor }\left(\frac{\mathrm{SCDRFWF}}{D_{T P S}}\right)} \\
& =\frac{594}{\text { floor }\left(\frac{594}{150}\right)}=198 \text { bytes }
\end{aligned}
$$

where $D T P S_{\text {ref }}$ means the DTPS that we want to use and DTPS is calculated packet size value nearest to $D T P S_{\text {ref }}$ that exactly fits to the sub-channel for given $\mathrm{N}$.

\subsection{Determination of Maximum Bandwidth Usage Upper Bound}

If the capacity of one sub-channel is not multiple of a data packet size, the last packet can not fit in the sub-channel and rest of the bandwidth will be wasted; e.g., for $\mathrm{N}=6$, we have 6 nodes and 6 of 30 sub-channels are used for voice packet transmissions and 1 or 2 sub-channels are used for video packet transmission in our system, remaining 22 or 23 (see eq.17) of 30 sub-channels are used for data packet transmissions. Hence; if $1 \mathrm{SC}$ is used by video packets at a frame, $23 \mathrm{SC}$ will be used for data packets of all system. Then Wasted Bandwidth by Data Transmission (WBDT) is calculated as in eq 19, choosing the packet size $=150$ bytes;

$$
\begin{aligned}
\text { WBDT } & =((594 \text { bytes })-(150 \text { bytes } 3)) \times 23 \\
& =3312 \text { bytes } / \text { frame }
\end{aligned}
$$

If the DTPS is used as 198 bytes as evaluated in eq. 18, then SCDRFWF calculated in eq. 15 will be able to carry 3 complete data packets without any bandwidth wastage. Note that, if voice packets came from other nodes also exist in the buffer, they can also share the same sub-channel together with other voice packets. However if the channel is not filled, the rest of the sub-channel bandwidth will again be wasted. Considering this state, the maximum possible data transmission via the whole channels can be calculated as in eq. 21. Since maximum theoretical hop count can at most be $\mathrm{N}-1$ hops for $\mathrm{N}=6$, each packet can stay in the network for at max 5 frames duration. As a result; totally 6 newly generated voice packets in the current frame with 6 nodes plus 6 voice packets 
generated in last 5 frames (totally $6+5 \times 6=36$ ) will be sent through the sub-channels in each frame by all nodes.

The total capacity of the full channel was given as 3564000 bytes/s in eq. 13. Since the system allocates a separate sub-channel for voice packets of each node in each frame, for $\mathrm{N}=6,36$ voice packets will be sent at max in each frame, totally $36 \times 10$ bytes will be transmitted by the nodes via 6 sub-channels allocated for voice packets of 6 nodes. That is; ( 6 SC x 594 bytes)-(10 bytes x 36 Voice packets $)=3204$ bytes will be wasted in a frame by the nodes. So, the wastage by voice packets in a second (WVCPIS) is calculated as;

$$
\begin{aligned}
\text { WVCPIS } & =(6 \times 594)-(10 \times 36)) \times 200 \\
& =640800 \mathrm{bytes} / \mathrm{sec}
\end{aligned}
$$

On the other hand when the nodes will use only 1 sub-channel for video conversation at 2 of every 4 frames, 23 sub-channels will be allocated for data packets and $1 / 2$ of 2 packet size (for last packet not fitting to sub-channel) will be wasted which equals to 1 sub-channel per 4 frames wastage or 0.25 packets/frame $\times 200$ frames $\times 198$ bytes = 9900 bytes. Since we select the size of video packets equal to SCC/sec with maximum quality that a sub-channel can handle as calculated in eq. 15, it will use the sub-channel completely and waste no bandwidth. As a result; the maximum possible successful bandwidth usage (MPSBU) that can be provided using given parameters and under given conditions, is evaluated by subtracting the total bandwidth wastage amount from MSU as in eq 21.

$$
\begin{aligned}
\text { MPSBU } & =3564000 \text { bytes }-640800 \text { bytes }-9900 \\
& =2913300 \text { bytes }
\end{aligned}
$$

\subsection{Calculation of the Size of Buffer}

Choosing the buffer size of the system is a critical point where choosing larger buffer sizes will require more memory for the nodes, allows more packets to be stored in the buffers and causes the packets to wait for longer durations in the buffer queues. On the other hand choosing smaller buffer sizes causes more packet losses. So it is necessary to calculate a considerable buffer size, which we will call "calculated buffer size".

Since the average number of hop counts evaluated by the simulation is AHC (Average hop counts) $\approx 3$, on average the packets of last 3 frames will stay in the buffers of the nodes in the network. Therefore totally $(\mathrm{N}) \times(\mathrm{AHC}+1) \times$ (number of packets generated by each node in a frame) packets will be in transmission during each frame. E.g., for $\mathrm{N}$ $=6$, buffer size of 264 (see eq. 24) slots will be enough provided that number hop counts for that instant is not greater than the calculated AHC of the system.

Calculated buffer size needed for data packets (CDBS) for our system can be evaluated theoretically as in eq. 22 and eq. 23 (for $\mathrm{N}=6, \mathrm{AHC}=3$ ) and graphically presented in Figure 1, for different number of AHC and number of nodes using the same spectrum.

$$
\begin{aligned}
C D B S & =S C D R F W F \times \text { number of allocated } S C \text { for data } \times(A H C+1) \\
& =(D S P F \times M A U \div 8) \times \text { number of allocated } S C \text { for data } \times(A H C+1) \\
& =(D S P F \times M A U \div 8) \times(N O S-N-1) \times(A H C+1) \text { bytes }
\end{aligned}
$$


It is assumed in eq. 22 that $\mathrm{N}$ sub-channels are used by voice packets of $\mathrm{N}$ nodes and 1 sub-channel is used by video packets at a time; so that (NOS-N-1) sub-channels will be allocated for data packets.

Since each generated packet group of a node will arrive to destination on AHC hops on average and since there will be a new generated packet group on each node at a frame, we multiply the resultant value in eq. 22 by $(\mathrm{AHC}+1)$.

$$
C D B S=(44 \times 108 \div 8) \times(30-6-2) \times(3+1)=52272 \text { bytes }
$$

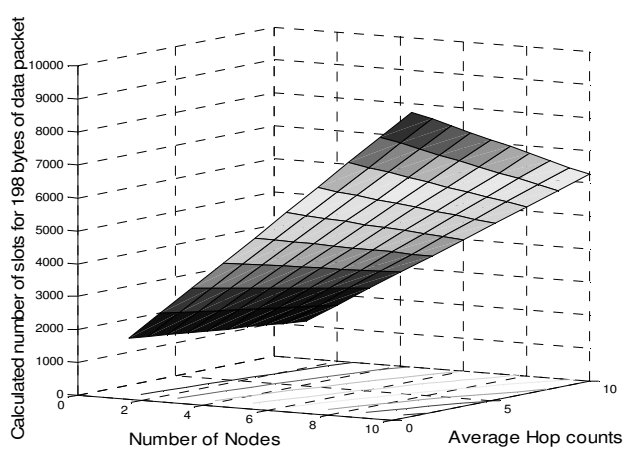

(a)

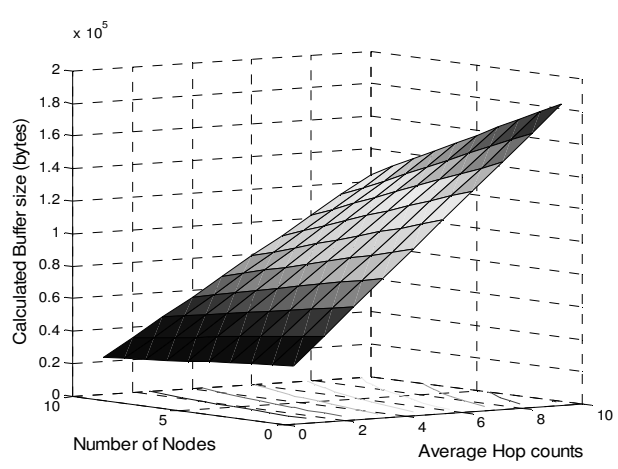

(b)

Figure 1. Calculated Buffer Sizes of 198 bytes data packets for a) number of slots, b) bytes, for different number of average hop counts and nodes sharing the spectrum.

Note that, it results with 78408 bytes and 396 buffer slots, by use of max hop count $(\mathrm{MHC})=5$, instead of AHC. Dividing CDBS to packet size, the calculated Number of Slots for Data packets (CNSDT) of each 198 bytes is evaluated as;

$$
\text { CNSDT }=52272 \text { bytes } / 198 \text { bytes }=264 \text { Slots }
$$

Calculated buffer sizes for voice and video buffers are also formulized in eq. 25 by multiplication of packet size by average number of all video packets in the buffers of all nodes in the network.

$$
\begin{aligned}
& \text { calculated video /voice Buffer Size }=(\text { packet size }) \times(V P S R) \times(N) \times(A H C+1) \text { bytes } \\
& \text { with }(V P S R) \times(N) \times(A H C+1) \text { slots }
\end{aligned}
$$

E.g., for $\mathrm{N}=6$, buffer size needed for voice packets can be calculated as in eq. 26 and buffer size needed for video packets can be calculated as in eq. 27 .

$$
\begin{gathered}
\text { Calculated voice Buffer Size }=(10) \times(1) \times(6) \times(3+1)=240 \text { bytes }(\mathrm{VPSR}=1) \\
\text { calculated video Buffer Size }=(594) \times\left(\frac{1}{4}\right) \times(6) \times(3+1)=3564 \text { bytes }=3.48 \mathrm{~kb} \\
\text { with }(\operatorname{VPSR}) \times(N) \times(\text { AHC }+1) \text { slots }(\mathrm{VPSR}=1 / 4)
\end{gathered}
$$

where VPSR is video/voice packet sending rate (1/4 for video packets and 1 for voice packets in our system), So that it has been provided that the total traffic management of all data types can be done using only the buffer size of 54648 bytes +240 bytes +3564 
bytes $=58452$ bytes $=57.08 \mathrm{~kb}$ and the total buffer size needed to manage the traffic is graphically presented in Figure 2 for various AHC and $\mathrm{N}$ values.

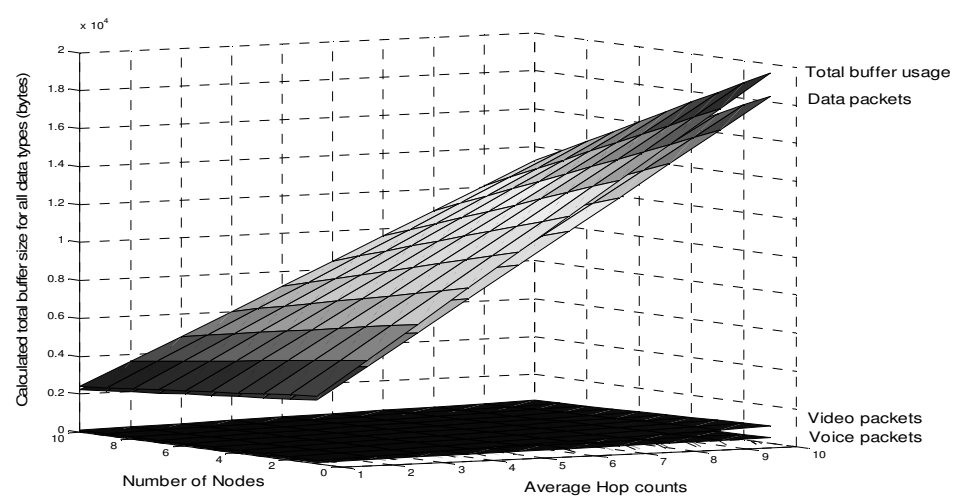

Figure 2. Calculated buffer sizes of data, voice, video packets and their sum for different $\mathrm{AHC}$ and different number of nodes sharing the spectrum.

\section{Cognitive APProaches for Maximized Network ThroughPUt}

In this system, the nodes manage the packets in their buffers by making decisions using the information they have about the state of the buffers of their next nodes towards their destination. The nodes in the network have random way point mobility model [8] with random speeds (from $25 \mathrm{~km} / \mathrm{h}$ up to $40 \mathrm{~km} / \mathrm{h}$ in our system). The loss ratio of the system is calculated as in eq. 28.

$$
\begin{aligned}
\text { loss ratio } & =\text { lost packets } / \text { sent packets } \\
& =\text { lost packets } /(\text { lost packets }+ \text { successfully sent packets })
\end{aligned}
$$

In regular OFDMA system, all the nodes have spectrum access to the sub-channels of the medium whenever they need. But assigning sub-channels for every node at the same time will not be possible in case of heavy packet traffic with limited number of subchannels. We suggest sharing the spectrum, by use of OFDMCAF system such that; the nodes, having buffers with more free slots, are assigned as receivers while the node whose buffer is nearest to get full, is assigned as transmitter. The transmitter uses the whole spectrum allocated for congested data type and frees its buffer at the current OFDMA frame. Thus, the packets will not wait in the buffers, the number of packet losses will be decreased and the data rate will be increased by the nature of adaptive rate algorithm which improves the throughput of the network.

\subsection{Cognitivity in Buffer Management}

\subsubsection{Route Reconstruction Algorithm}

We need a good dynamic spectrum sharing technique to transport as more packets as possible in unit of time and free the buffers. So the packets should arrive to final destination by minimum number of hops and with minimal delay amounts. Since the nodes in the network always change position, each node recalculates and updates the path of each packet and determines the next node towards the destination using the Fastest Path algorithm in [6]. 
Making these calculations for benefit of other nodes is part of the cooperation; the route recalculation algorithm is also activated in all cases of our study for providing a fair comparison among all.

\subsubsection{Adaptive data rate (AR) algorithm}

When congestion and packet losses occur in the network, the transmission rate is decreased using adaptive data transmission algorithm. This method adds learning and experience features to the network where the nodes adapt to changing network conditions. It is also shown in [9] that adaptive rate has a positive effect on the system throughput. Since we have network conditions with heavy packet traffic, the maximum data packet generation/transmission rate using AR, can be calculated. In eq. 29, Maximum data rate per node (MDRPN) in a frame is evaluated by calculating the total bandwidth (MSU) per SC used by data packets (TSFDT/NOS) per node $(1 / \mathrm{N})$ per frame ( $1 / \mathrm{FPS}=\mathrm{FL})$ and multiplying the resultant value by the rate of successful packet transmission.

$$
\text { MDRPN }=\text { MSU }(\text { bytes } / \mathrm{s}) \times \frac{T S F D T}{N O S} \times \frac{1}{N} \times(F L) \times \frac{\text { Succesfully sent } p .}{\text { Succesfully sent } p .+ \text { lost } p}
$$

For the case that MSU=3564000 bytes/s, NOS=30 SC, N=6 nodes, FL= $5 \mathrm{~ms}$ and there is no packet loss; if we have two nodes transmitting video packets, we have TSFDT = 22 and MDRPN = 2178 bytes/node (11 packets/node), if we have one node transmitting video packets, we have TSFDT $=23$ and $\mathrm{MDRPN}=2277$ bytes/node $(11.5 \approx 11$ data packets/node) using eq. 29 . Note that all the results in the simulation are dynamically calculated and updated by making calculation according to the parameter values and network state at that instant. It also generalizes for any number of nodes, any VPSR value and for any other parameter set used in the system.

\subsubsection{Buffer Management by Queue Optimization (BM)}

If a node starts to send its packets, it determines the next nodes of each packet going towards their final destinations using the path reconstruction algorithms. It resorts its buffer such that the packets with same destinations are grouped to be sent together and the packets whose next node has more free memory will be sent first according to dynamic spectral aids algorithm. This process continues during the current OFDMA frame as long as no packet loss occurs and as soon as the transmitting node still has packets to send.

\subsection{Dynamic Spectral Aids (Sa) Algorithm}

In our system N (number of nodes) of NOS (number of sub-channels) sub-channels are allocated to voice packets of $\mathrm{N}$ nodes (one for each) and N/4 or N/4+1 sub-channels are allocated to video packets of the nodes which want to send video packets through the sub-channel. The rest of the sub-channels are allocated to the node which gives alert of fuller buffer most. If a node starts to transmit packets, it makes buffer optimization and transmits the packets whose calculated next node buffer has the largest free memory. If the buffer of freest node is also full, the transmitter loses its first trial packet of chosen destination and gives its turn to the node who gave alert of the 'spectrum need' most. 
The spectrum usage turn is again allocated to the node which most needs the spectrum at the end of the frame.

\section{ASYMPTOTIC THROUGHPUT ANALYSIS OF UNICAST TRANSMISSION}

In our system the Base Station (BS) is considered to use an Omni-directional antenna to transmit packets to all relay nodes in the cellular area. Since the user which has worst conditions determines the total system throughput performance, usage of unicast scheme may be useful in case the best user is chosen all the time [10]. The resultant formulation of the works done in [10] that evaluate the throughput of a unicast system by asymptotic analysis is given in eq. 30 . It will also be divided by $\mathrm{N}$ in order to evaluate the unicast throughput per user.

$$
\begin{aligned}
& \mathrm{Ru}=B \log _{\mathrm{a}}\left(1+\frac{\rho_{\mathrm{0}} \ln (N)}{d_{e} n}\right)+\frac{B n}{2 \ln (2)} \\
& \text { where } \rho_{\mathrm{0}}=\frac{P}{N_{\mathrm{a}}} \times K \times \beta
\end{aligned}
$$
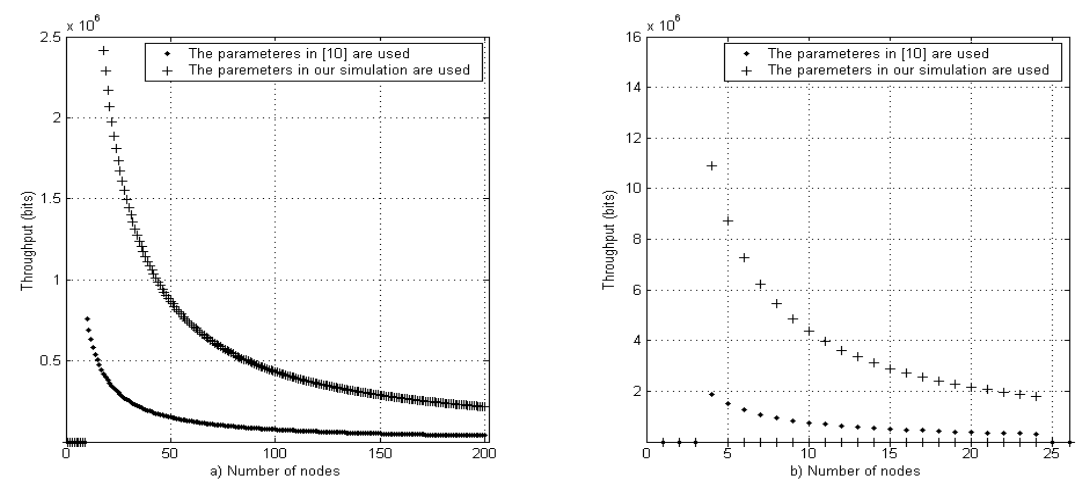

Figure 3. The throughput results (bits/sec) of eq. 30 for the parameters used in [10] and used in our simulator for increased number of nodes a) from 10 to 200 as in [10] and b) from 4 to 24 as in our system.

The results evaluated by eq. 30 for the parameters used in [10] and for the parameters used in our simulation are illustrated in Figure 3.a, Figure 3.b. and Figure 12 for confirmation. Since there are 30 sub-channels in our system and since each of $\mathrm{N}$ node will use 50 sub-channels in every 200 frames (with $\mathrm{VPSR}=1 / 4$ ) using a separated subchannel for its own voice conversation; [NOS- $(\mathrm{N}+\mathrm{N} / 4)] / \mathrm{N}$ sub channels will be used by $\mathrm{N}$ nodes in the cell for which [NOS-(N+N/4)]/N $>=0$ and $\mathrm{N}<=24$. That's why $\mathrm{N}$ is increased up to 24 in our simulation. The results evaluated for the activation cases of, "none of the methods", "adaptive rate", "adaptive rate plus buffer management" and "Adaptive Rate plus Buffer Management plus Spectral Aids" are presented in Figure 12.

\section{Performance evaluation}

\subsection{Performance Evaluation of Cognitive Methods on Throughput Performance for Fixed Number of Nodes $(\mathrm{N}=6)$}

At each simulation run, the improvements made on; efficient bandwidth use, data packet loss and throughput of the system in different conditions, are simulated with and without applying each cognitive method to the system, by not taking one of the 
algorithms into progress, we have been able to separately observe the improvement and the effects of each algorithm on system performance.
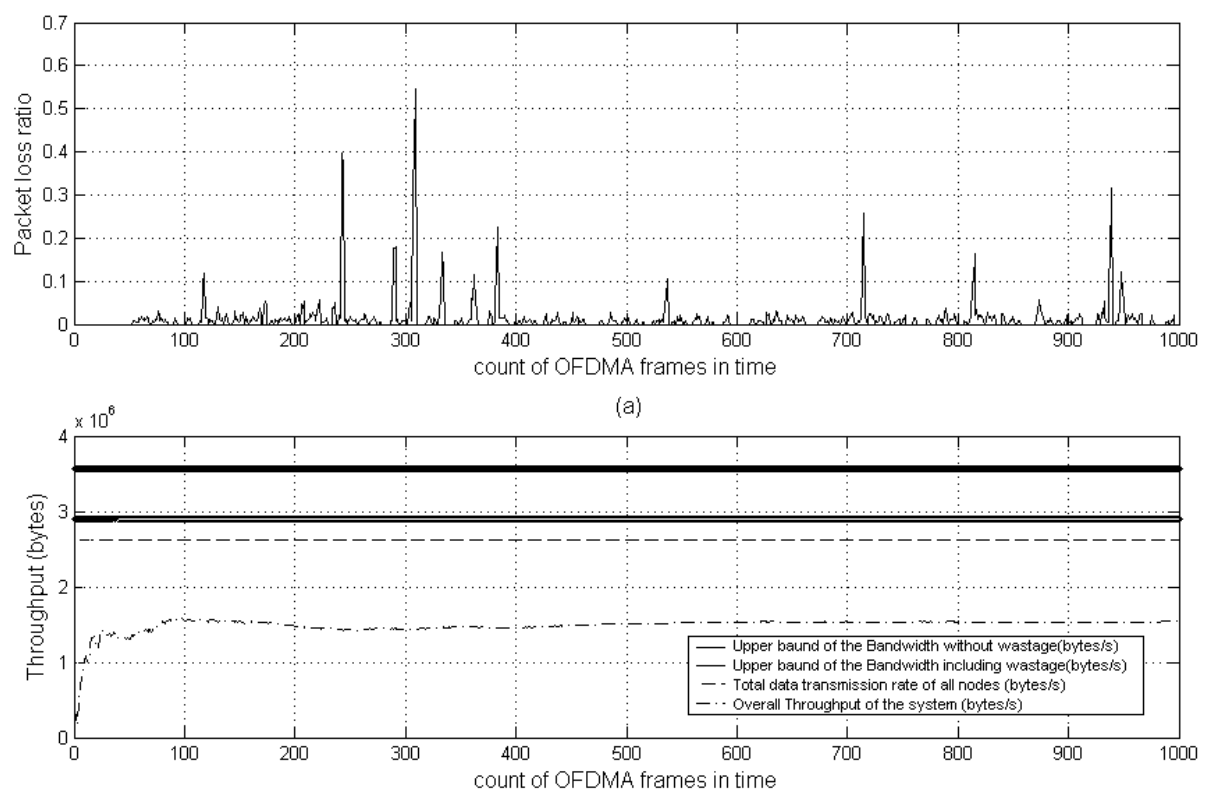

(b)

Figure 4.(a) Packet loss ratio of the whole network in last period of 3 frames (b) bandwidth upper bound, data packet generation rate and throughput of the pure system vs. frame count, without usage of any novel algorithm.

Figure 4 illustrates the pure system simulation results when none of the methods is activated and the system behaves under normal conditions. For $\mathrm{N}=6$, the simulation output graphics of some cases are shown on Figures 4-7 and numeric values of sent/lost packets, spectral use and throughput amounts taken from the simulation results for all cases, are listed in Tables 2 and 3.

The throughput values evaluated by the simulation and shown on Table 3 can also be checked using the number of successfully sent packets simulation outputs on Table 2 such that;

$$
\text { Throughput }(\text { bytes / sec })=\frac{\left(\begin{array}{l}
\text { data packet size } \times \text { number sent data packets } \\
\text { +voice packet size } \times \text { number sent voice packets } \\
\text { +video packet size } \times \text { number sent video packets }
\end{array}\right)}{T_{\text {sim }}(\mathrm{sec} .)}
$$

If larger buffer size were used, the packet loss ratio would decrease with respect to smaller buffer sizes and it would have positive effect on the throughput. It's seen on Figure 10 that packet loss ratio is decreased by using larger buffer size with respect to selecting the calculated one. But Figure 9 indicates that the throughput of the system is not improved by using large buffer size, because larger buffers store and hold more packets inside and cause the packets to wait for longer durations in the buffers. 
When the adaptive rate algorithm is activated, the total data transmission rate of all nodes adaptively varies according to congestions and packet losses occurring in the network in the last period as described in [9]. This variation can be read from the upper dashed line in Figures 5.b, 6.b and 7.b. The lines on Figure 5.b, Figure 6.b and Figure 7.b which illustrate the packet generation/transmission rate are always symmetrical with the corresponding packet loss rates shown on Figure 5.a, Figure 6.a and Figure 7.a.

Table 2. The number of sent/lost packets results of simulations for application conditions of the methods during 1000 OFDMA frames (5 seconds).

\begin{tabular}{|c|c|c|c|c|c|c|c|c|c|c|c|}
\hline \multirow{2}{*}{$\begin{array}{c}\text { Simulation Output } \\
\text { Data } \\
(\mathrm{N}=6)\end{array}$} & \multicolumn{3}{|c|}{$\begin{array}{l}\text { End to end successful } \\
\text { Average transmit time } \\
\text { of a packet (ms) }\end{array}$} & \multicolumn{2}{|c|}{ 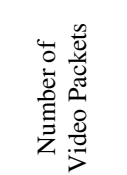 } & \multicolumn{2}{|c|}{ 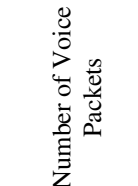 } & \multicolumn{2}{|c|}{ 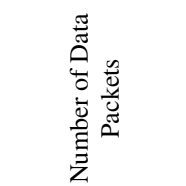 } & \multicolumn{2}{|c|}{ 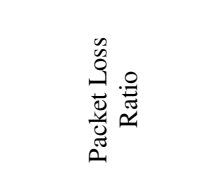 } \\
\hline & $\sum_{i}^{8}$ & $\frac{0}{0}$ & صีّ & $\begin{array}{l}\vec{\Xi} \\
\ddot{D}\end{array}$ & 苛 & $\begin{array}{l}\vec{\Xi} \\
\ddot{D}\end{array}$ & 岁 & $\begin{array}{l}\vec{\Xi} \\
\ddot{D}\end{array}$ & 范 & 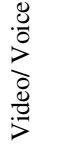 & $\stackrel{\widetilde{J}}{\tilde{\Xi}}$ \\
\hline No methods & $\begin{array}{c}10,1 \\
2\end{array}$ & 2,09 & 0,17 & 494 & 0 & 2389 & 0 & 29344 & 4456 & $0.00 \%$ & $13.18 \%$ \\
\hline All - adaptive rate & 9,86 & 1,94 & 0,14 & 507 & 0 & 2576 & 0 & 35953 & 653 & $0.00 \%$ & $1.78 \%$ \\
\hline All +larger buffer & 9,71 & 1,97 & 0,14 & 515 & 0 & 2532 & 0 & 36691 & 58 & $0.00 \%$ & $0,16 \%$ \\
\hline $\begin{array}{l}\text { All - buffer } \\
\text { management }\end{array}$ & 9,67 & 1,94 & 0,15 & 517 & 0 & 2582 & 0 & 33978 & 654 & $0.00 \%$ & $1.89 \%$ \\
\hline All - spectral Aid & 9,96 & 1,94 & 0,16 & 502 & 0 & 2575 & 0 & 31419 & 786 & $0.00 \%$ & $2,44 \%$ \\
\hline All + Calc. Buffer & 9,71 & 1,97 & 0,13 & 515 & 0 & 2544 & 0 & 37631 & 182 & $0.00 \%$ & $0.48 \%$ \\
\hline
\end{tabular}

Table 3. The results of simulations for application conditions of the methods during 1000 OFDMA frames

\begin{tabular}{|c|c|c|c|c|c|c|}
\hline $\begin{array}{l}\text { Simulation Output } \\
\text { Data } \\
(\mathrm{N}=6)\end{array}$ & $\begin{array}{l}\text { Average } \\
\text { Data } \\
\text { Packet } \\
\text { Loss } \\
\text { Ratio }\end{array}$ & $\begin{array}{l}\text { Average } \\
\text { Effective } \\
\text { Bandwidth } \\
\text { usage in } \\
2913300 \\
\text { bytes/sec } \\
\text { (bytes/s) }\end{array}$ & $\begin{array}{c}\text { Average } \\
\text { Effective } \\
\text { Bandwidth } \\
\text { usage } \\
\text { percentage in } \\
2913300 \\
\text { bytes/sec } \\
\text { (bytes/s) }\end{array}$ & $\begin{array}{c}\text { Average } \\
\text { overall } \\
\text { Throughput } \\
\text { Provided } \\
\text { ( bytes/s) }\end{array}$ & 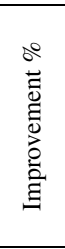 & $\begin{array}{l}\text { Improvement Loss } \\
\text { of the Throughput } \\
\text { by not applying } \\
\text { the algorithm (i.e. } \\
\text { improvement of } \\
\text { the algorithm) }\end{array}$ \\
\hline No methods & $13.18 \%$ & 2642358 & $90 \%$ & 1225488 & $0 \%$ & $27 \%$ \\
\hline All - adaptive rate & $1.78 \%$ & 2790893 & $95 \%$ & 1489122 & $22 \%$ & $5 \%$ \\
\hline All - spectral Aid & $2.44 \%$ & 2782438 & $95 \%$ & 1308980 & $7 \%$ & $20 \%$ \\
\hline $\begin{array}{l}\text { All - buffer } \\
\text { management }\end{array}$ & $1.89 \%$ & 2751435 & $94 \%$ & 1412112 & $15 \%$ & $12 \%$ \\
\hline All +larger buffer & $0.16 \%$ & 2808181 & $96 \%$ & 1519210 & $24 \%$ & $3 \%$ \\
\hline All + opt. Buffer & $0.48 \%$ & 2801605 & $96 \%$ & 1556458 & $27 \%$ & $0 \%$ \\
\hline
\end{tabular}



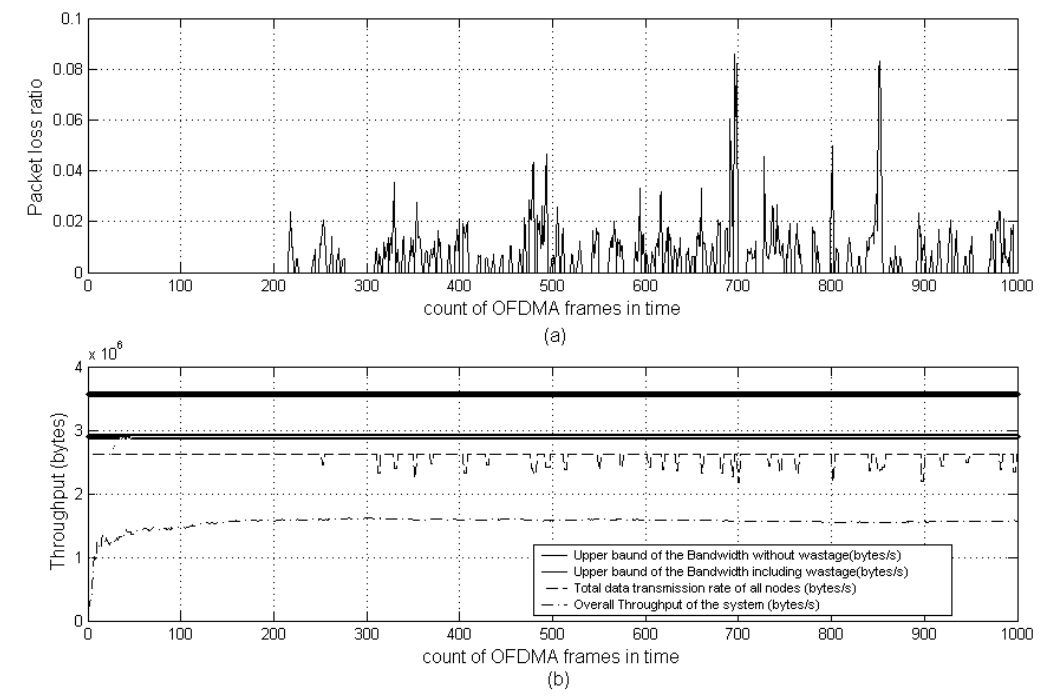

Figure 5. (a) Packet loss average of the whole network in last period of 3 frames (b) bandwidth upper bound, data packet generation rate and throughput of the system vs. frame count when all methods are active and using larger buffer size.

On the other hand, deactivating the use of buffer management algorithm, allows more congestions on the buffers of the nodes and more packet losses occur. As it is observed from Figure 8, the number of sent voice / video packets has approximately same results for each case. Because, a separate sub-channel is allocated for real time voice and video packets of each node at each OFDMA frame. Their buffers are not overloaded by the system and no voice or video packet loss exists during the transmission. Thus, system throughput performance is determined by the number of sent/lost packets of high traffic loaded data packets shown in Figure 8 which differs for each algorithm.
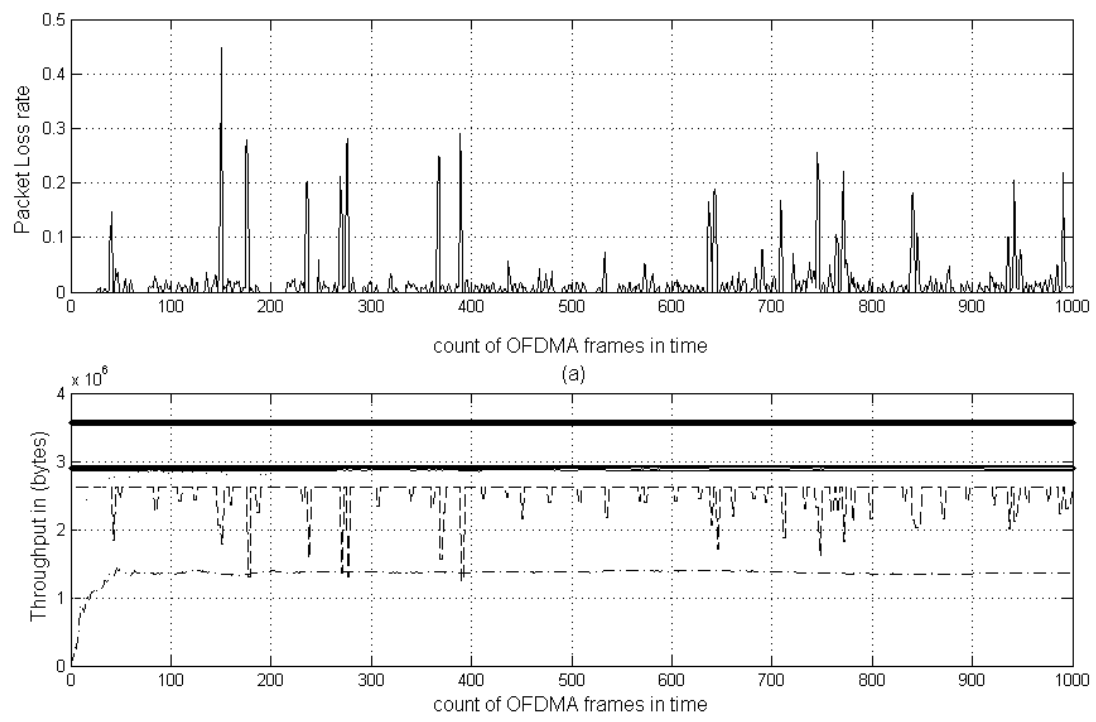

(b) 
Figure 6. (a) Packet loss average of the whole network in last period of 3 frames (b) bandwidth upper bound, data packet generation rate and throughput of the system vs. frame count, without usage of cognitive Dynamic Spectrum Aids method.

As shown on Figure 9, when all cognitive methods are activated, the throughput is maximized up to 1556458 bytes, using all the novel cognitive methods at the same time. It is also seen in Figure 9 that, the greatest improvement is supplied by the spectral aids algorithm. That's why the system average throughput performance is reduced most during the absence case of SA algorithm.
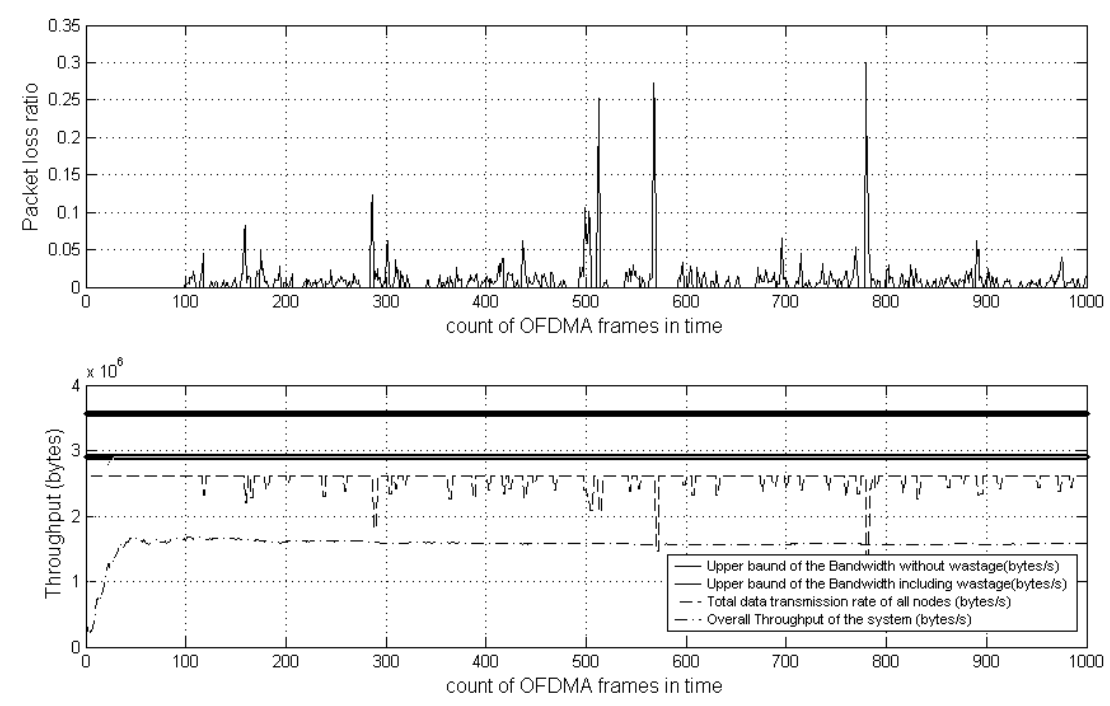

Figure 7. (a) Packet loss average of the whole network vs. frame count in last period of 3 frames (b) bandwidth upper bound, data packet generation rate and throughput of the system vs. frame count, with usage of all cognitive methods and calculated buffer size.

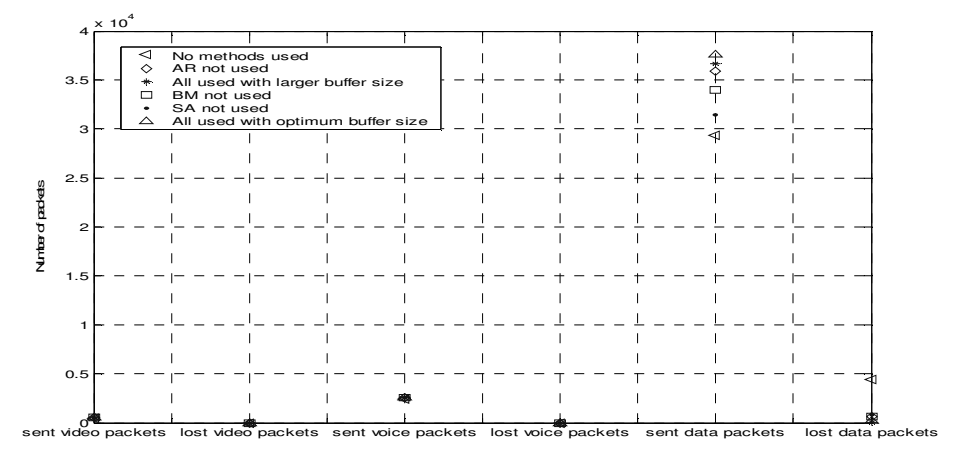

Figure 8. The improvement amounts of the methods for number of video/voice and data packets successfully transmitted to the final destination in 1000 frames, using Fastest Path routing algorithm. 
International Journal of Wireless \& Mobile Networks ( IJWMN ), Vol.2, No.3, August 2010

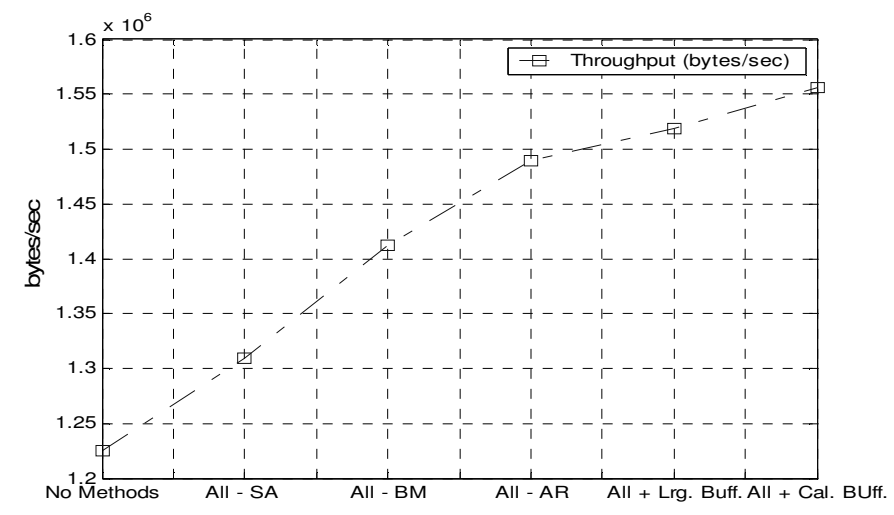

Figure 9. Throughput improvement on OFDMCAF by use of novel methods

One of the most important criteria that influence the throughput of the system is efficient usage of spectrum and decreasing the packet loss rate. The average spectrum usage rates of the successfully sent packets are seen on Figure 11. It is seen that the most effective spectrum usage is provided when all proposed methods are activated.

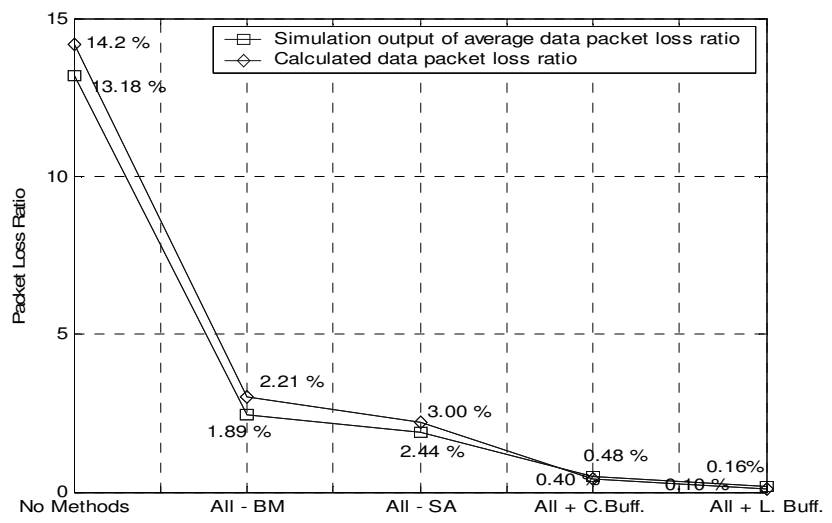

Figure 10. Average data packet loss ratio of the system by use of different methods and confirmation of the simulation results with the results evaluated using eq.. 33-36

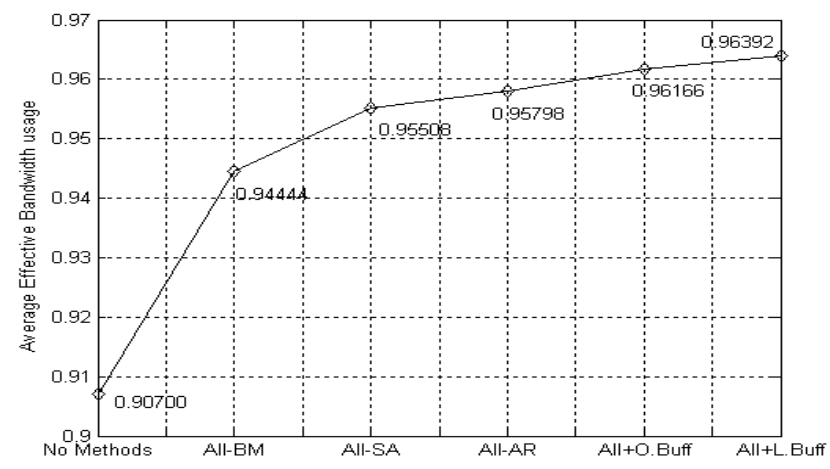

Figure 11. Average Effective Spectral Usage of the system, by using the novel cognitive methods. 
When total number of packets in the network is assumed to be distributed to the nodes proportional to waiting durations of the nodes, such that the one just used the spectrum has thought to have the emptiest buffer. The average packet loss probability for rest of $\mathrm{N}-1$ nodes (excluding the current transmitter and the packets it has) calculated using eq. 32 and eq. 33, gives the resultant average packet loss ratio.

Our results in Figure 10 satisfy this condition by enabling the new proposed algorithms. Let the packet distribution rates of the nodes to be;

$$
\text { Node }_{1} \rightarrow 1, \text { Node }_{2} \rightarrow 2, \ldots, \text { Node }_{N-1} \rightarrow N-1, \text { Node }_{N} \rightarrow N
$$

where, Node ${ }_{1}$ is considered to be the one just transmitted its packets and Node $_{N}$ is considered to be the current transmitter with the fullest buffer. Then, the probability of loosing a packet in the Node $_{\mathrm{n}}$ can be calculated as;

$$
\begin{aligned}
& P_{\text {lost }}(n)= \frac{\frac{\text { Packet Dist.rate of the Node }_{n}}{\text { Sum of Dist.rates of all nodes }} \times \text { Total Packet count }}{\text { Buffer size }} \\
&= \frac{\frac{(n)}{N \times(N+1)} \times \text { Total Packet count }}{2} \\
& P_{\text {lost }}(n)=\frac{2 \times(n) \times \text { Total Packet count }}{\text { Buffer size } \times N \times(N+1)}
\end{aligned}
$$

Remembering that, we assume to have total 264 data packets on average in all nodes buffers and we equalize the number of slots in data buffer, to this value (see eq. 24), let the packets to be distributed to 6 nodes according to their waiting durations as;

$$
\begin{aligned}
& \text { Node }_{1} \rightarrow 1(12 \text { packets }), \text { Node }_{2} \rightarrow 2(25 \text { packets }), \text { Node } e_{3} \rightarrow 2(38 \text { packets }), \\
& \text { Node }_{4} \rightarrow 2(50 \text { packets }), \text { Node }_{5} \rightarrow 2(64 \text { packets }), \text { Node }_{6} \rightarrow 2(75 \text { packets }), \\
& \text { Totally } \rightarrow 264 \text { packets }
\end{aligned}
$$

Then the probability of a packet to get lost in any of the nodes can be evaluated by calculating the probability of sending packet to a node (with sending probability of 1 / $(\mathrm{N}-1)$ excluding the sender itself) and loosing the packet there, as;

$$
P_{\text {loss }}=\frac{1}{N-1} \times P_{\text {lost }}(1)+\frac{1}{N-1} \times P_{\text {los }} t(2)+\ldots+\frac{1}{N-1} \times P_{\text {lost }}(N-1)
$$

Then using $\mathrm{P}_{\text {lost }}(\mathrm{n})$ from eq.32, we have the resultant formula as in eq. 33.

$$
P_{\text {loss }}=\frac{1}{(N-1)}\left(\sum_{n=1}^{N-1} P_{\text {lost }}(n)\right)
$$

For $N=6$, Buffer size $=264$ and Total Packet count $=264$, it results with, 
International Journal of Wireless \& Mobile Networks ( IJWMN ), Vol.2, No.3, August 2010

$$
\begin{aligned}
P_{\text {loss }}= & \frac{1}{N-1} \sum_{n=1}^{N-1} \times P_{\text {lost }}(n) \\
& =\sum_{n=1}^{N-1} \frac{1}{N-1} \times \frac{2 \times(n) \times \text { Total Packet count }}{\text { Buffer size } \times N \times(N+1)} \\
& =\sum_{n=1}^{N-1} \frac{1}{6-1} \times \frac{2 \times(n) \times 264}{264 \times 6 \times(6+1)} \\
& =\frac{1}{5} \times\left[\frac{1}{21}+\frac{2}{21}+\frac{3}{21}+\frac{4}{21}+\frac{5}{21}\right] \\
& =0.142=\% 14.2
\end{aligned}
$$

And it results with $\mathrm{P}_{\text {loss }}=2.5 \%$ with buffer size of 500 slots. The calculated result also matches with the simulation results presented in Table 3 and Figure 10.

If the proposed Buffer Management (BM) technique is activated, the packet loss occurs if and only if all the packets in the buffer of the transmitting node have the next nodes with full buffer. I.e., sum of probability for having packets to any possible next node combination will be multiplied by the probability of loosing the packet there.

$$
\begin{gathered}
P_{\text {loss } \_B M}=\frac{\left(\begin{array}{l}
N-1 \\
1
\end{array}\right) p^{1}+\left(\begin{array}{l}
N-1 \\
2
\end{array}\right) p^{2}+\left(\begin{array}{l}
N-1 \\
3
\end{array}\right) p^{3}+\ldots+\left(\begin{array}{l}
N-1 \\
N-1
\end{array}\right) p^{N-1}}{\sum_{n=1}^{N-1}\left(\begin{array}{l}
N-1 \\
n
\end{array}\right)} \\
P_{\text {los } \_B M}=\frac{\sum_{n=1}^{N-1}\left\{\left(\begin{array}{l}
N-1 \\
n
\end{array}\right) \times p^{n}\right\}}{\sum_{n=1}^{N-1}\left(\begin{array}{l}
N-1 \\
n
\end{array}\right)}
\end{gathered}
$$

For $\mathrm{N}=6$, we have,

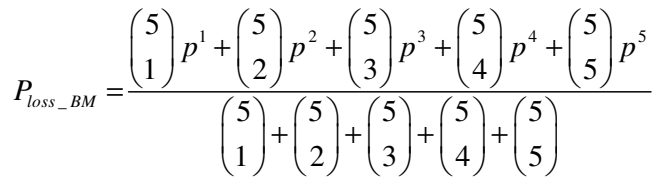

$$
\begin{aligned}
& P_{\text {loss } \_B M}=\frac{5 p^{1}+10 p^{2}+10 p^{3}+5 p^{4}+p^{5}}{5+10+10+5+1} \text {, for } p=0.142 \\
& P_{\text {loss_BM }}=0.03=3.00 \%
\end{aligned}
$$

from eq. 34 which also corresponds and matches with the result of All - spectral Aids in Table 2 and Table 3 and All-SA on Figure 10.For the case of applying SA algorithm without BM algorithm (All - Buffer Management in Table 3) the packet loss occurs if and only if we have no packet belongs to a node having free slot in its buffer. Being aware of that, if there are nodes with full buffer in the network, it means that, the buffer of transmitter is already full (so it took the spectrum usage turn), taking account the buffer state combination of rest of $\mathrm{N}-1, \mathrm{P}_{\text {loss_SA }}$ is calculated by sum of Probabilities for;

- Only the transmitter has full buffer, others not, choosing a full buffer (prob.=0)+

- 2 nodes with full buffer, N-2 not and choosing the full one +

- 3 nodes with full buffer, N-3 not and choosing one of the nodes with full buffer+

- $\quad \ldots$ 
- $\mathrm{N}($ all) nodes are full, 0 not, choosing a full buffer (prob.=1),

So, for remaining N-1 nodes (excluding the transmitter itself), we have;

$$
\begin{gathered}
P_{\text {loss_SA }}=\left(p^{1} \times(1-p)^{(N-1)-1} \times \frac{1}{N-1}\right)+\left(p^{2} \times(1-p)^{(N-1)-2} \times \frac{2}{N-1}\right)+\ldots+\left(p^{n} \times(1-p)^{(N-1)-(n)} \times \frac{n}{N-1}\right) \\
P_{\text {loss_SA }_{-}}=\sum_{n=1}^{N-1} P^{n} \times(1-\mathrm{p})^{(\mathrm{N}-1)-\mathrm{n})} \times \frac{n}{N-1}
\end{gathered}
$$

where $\mathrm{p}$ values in eq. 34 and eq. 35 are used as depicted in eq. 33. For $\mathrm{N}=6$ and $\mathrm{p}=$ $14.2 \%$ we have $\mathrm{P}_{\text {loss_SA }}=2.21 \%$ from eq. 35 which also corresponds and matches with the result of All - Buffer Management in Table 3 and All-BM on Figure 10. When we apply both BM and SA algorithms, we have eq. 36 by combining eq. 34 and eq. 35 .

$$
P_{\text {loss_rate } A L L}=\sum_{n=1}^{N-1}\left(\frac{\left(\begin{array}{l}
N-1 \\
n
\end{array}\right) \times p^{n}}{\sum_{r=1}^{N-1}\left(\begin{array}{l}
N-1 \\
r
\end{array}\right)} \times(1-p)^{((N-1)-n)} \times \frac{n}{N-1}\right)
$$

For $\mathrm{N}=6$ and using 264 slots (calculated buffer size) in the buffer, we have $\mathrm{P}_{\text {loss_ALL }}=$ $0.40 \%$ from eq. 36 and we have $\mathrm{P}_{\text {loss_AL }}=0.10 \%$ using 500 slots (larger buffer size) which also respectively corresponds and matches with the results of All + Cal. Buffer and All + larger buffer in Table 3 and All-C. Buff., All + L. Buff on Figure 10.

\subsection{Performance evaluation and confirmation of the throughput results of novel cognitive methods for varied number of nodes (from 4 ranging up to 24)}

It's seen from Figure 12 that, when none of the novel methods of OFDMCAF is activated, OFDMCAF simulation results and the pure OFDMA simulation results match with the results of asymptotic throughput analysis made in [10] for large $\mathrm{N}$ values as depicted in [10]. The simulation results of OFDMCAF with only BM (buffer management) and with both BM and SA (spectral aids), illustrate that the throughput of the system is improved by activation of the novel proposed methods of OFDMCAF. It is observed that the throughput curves for all methods shown on Figure 12 approach each other by the increased number of nodes because of the increased number of used real time video and voice sub-channel by increase of node count and decreased number of remaining sub-channels used for data transmission. The unicast system throughput performance analysis is done using the extreme value theorem in [10] but the bandwidth wastage caused by unfilled sub-channels is not considered there. In eq. 37 the throughput amount is calculated ignoring the bandwidth wastage amount. The resultant throughput values are multiplied by average hop count value (taken as 3 hops) to see total successful spectral usage for unicast analysis, simulation runs and calculations for fairness. The same parameter values are used for asymptotic unicast analysis results in Figure 12 and Figure 3 as they are used in our simulation as; path loss constant $\mathrm{K}=10^{\circ}$ $(0 \mathrm{~dB}), \mathrm{dc}=50 \mathrm{~m}$, path loss exponent $\mathrm{n}=2$ for free space, $\mathrm{SNR}=10^{3}$ and $\mathrm{B}=10 \mathrm{MHz}$, where $\mathrm{K}=10^{3.15} \mathrm{dc}=1000 \mathrm{~m}, \mathrm{n}=3.5$ and $\mathrm{B}$ is taken as $1 \mathrm{MHz}$ for unicast analysis made in [10] and shown in Figure 3 and Figure 12. 


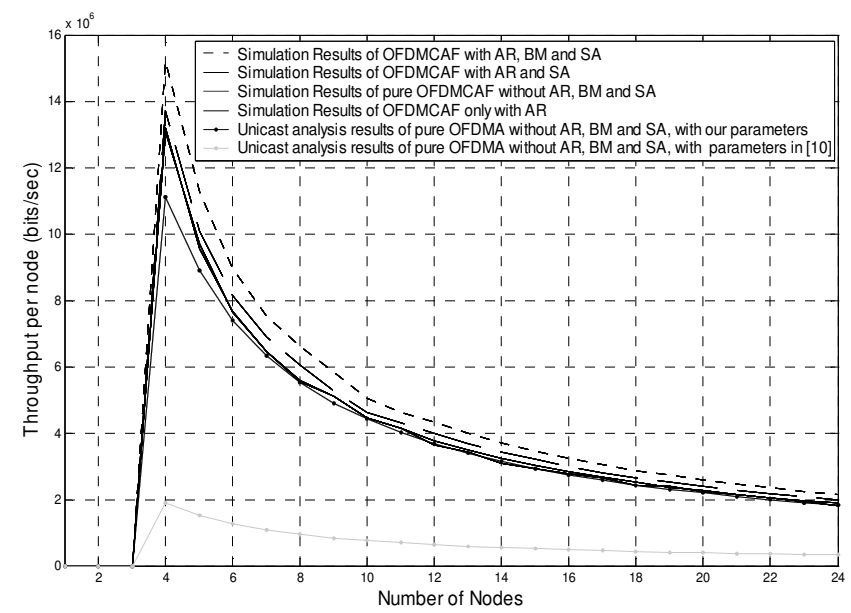

Figure 12. The simulation results of OFDMA,OFDMAF and unicast asymptotic analysis [10] for throughput per node performance improvement in cases of applying adaptive rate(AR), AR plus buffer management(BM) and AR plus BM plus spectrum sharing (SS), using Fastest Path routing algorithm.

The throughput calculation ignoring the bandwidth wastage is evaluated as in eq.37.

$$
T H R_{\text {without wastage }}(\text { bits }) \square \frac{\left(\left(T H R_{\text {with wastage }}(\text { bytes })+\text { wastage }(\text { bytes })\right) \times 8 \times A H C\right)}{N}
$$

By a simple check for $\mathrm{N}=6$ nodes and for "no method is active" case; since we have system throughput $=1225488$ bytes as in Table 3 and bandwidth wastage $\approx 640800$ bytes from eq. 20, we have the result in eq. 38 for $\mathrm{AHC}=3$,

$$
T H R_{\text {without wastage }} \square \frac{(1225488+640800+9900) \times 8 \times 3}{6}=7504752 \text { bits }
$$

The value in eq. 38 can also be confirmed by reading 7638500 bits from the simulation results on Figure 12 for the case $\mathrm{N}=6$.

\section{CONCLUSION}

In this study, the throughput per node of a MANET (Mobile Ad-Hoc Network) is improved by the novel proposed algorithms where adaptive rate algorithm is also used. It is also shown that the packet loss ratio is decreased by use of novel methods and the system throughput is increased with increasing packet generation rate caused by decreasing packet loss ratio. Efficient spectrum sharing is provided applying the proposed methods to the new form of OFDMA and TDMA which we call as OFDMCAF and doesn't require complex modifications on the current frame and system structure. On the other hand the calculated buffer size for different number of average hop counts and different number of nodes is also formulized and calculated. It's validated by the simulation outputs that, using larger buffer size can not improve the throughput even it reduces the packet loss ratio. The throughput improvements by each method has been separately analyzed for fixed $(\mathrm{N}=6)$ and variable number of nodes (from 4 ranging up to 24). Calculation of packet losses for each novel proposed algorithm is probabilistically formulized. The results evaluated by the simulation are 
confirmed by these calculations and by comparing the throughput results with the results evaluated in the literature. Finally, the confirmed system performance improvements are graphically illustrated. The algorithms, formulizations and results of the analysis can be used in designing unicast mobile multimedia ad-hoc network with decreased packet loss rate and improved throughput.

\section{References}

[1] Amitabh K., "Mobile broadcasting with WIMAX", Book, Elsevier Inc., 2008.

[2] Mobile WIMAX- Part II: “A Comparative Analysis”, Copyright WIMAX Forum , 2006

[3] Mobile WIMAX- Part I: "A Technical Overview and Performance Evaluation Prepared on Behalf of the WIMAX Forum", Copyright WIMAX Forum, 2006.

[4] Preveze B. and Şafak A., "Throughput Maximization of Different Signal Shapes Working on 802.16e Mobile Multi-hop Network Using Novel Cognitive Methods", Recent Trends in Wireless and Mobile Networks (WIMO 2010), Vol.84, pp. 71-86, 2010

[5] Link: http://en.wikipedia.org/wiki/Cognitive_radio. Link last access date: July 10th 2010.

[6] Yi X. and Wanye W., "Finding the Fastest Path in Wireless Networks", IEEE International Conference on Communication (ICC 2008), pp. 3188-3192, 2008.

[7] L. Huang; M. Rong; L. Wang; Y. Xue, Schulz, E. "Resource Allocation for OFDMA Based Relay Enhanced Cellular Networks", IEEE 65th VTC 2007 ,pp. 3160 - 3164 ,2007.

[8] Deborah E., Daniel Z., Li T., Yakov R., and Kannan V., "Source : Demand Routing:Packet format and forwarding specification", Internet RFCs archive, (version 1)., pp. 1-7, 1995.

[9] Iannone L and Fdida S, "Can Multi_rate Radios reduce end_to_end_delay in mesh network? A simulation case study", Mesh Networking: Realizing the Wireless Internet (Meshnets'05), 2005.

[10] T. Girici, "Asymptotic throughput analysis of multicast transmission schemes" Int. J. Electron. Commun. (AEÜ) 63 pp. 901-905,2009

\section{Authors}

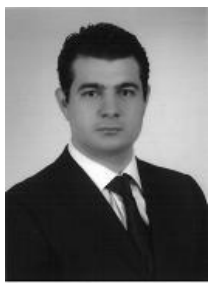

Barbaros Preveze received the B.S. and M.S. degrees from Çankaya University, Ankara,Turkey, in 2001 and 2004, respectively $\mathrm{He}$ is a Ph.D. student at Başkent University, Ankara since 2004. He is working as a research assistant at Çankaya University since September 2001. His research interests are in computer communications, wireless communications and networking, with a current focus on throughput improvement of multihop wireless networks.

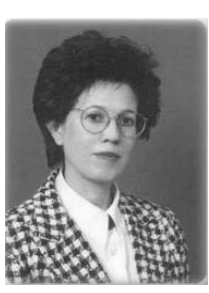

Aysel Şafak received the B.S. of Physics Engineering from Hacettepe University ,Ankara, Turkey in 1975, M.S. and PHd. degrees from Electronics Engineering department of Delft technical University, in 1987 and 1994, respectively. She is currently an Assistant Professor in Başkent University, Ankara, Turkey. Her research interests are in mobile communications, modeling of communication channels, communication systems, OFDM and MIMO systems. 